\title{
Acanthamoeba encystment: multifactorial effects of buffers, biocides, and demulcents present in contact lens care solutions
}

This article was published in the following Dove Press journal:

Clinical Ophthalmology

13 October 2015

Number of times this article has been viewed

\section{Christopher J Kovacs \\ Shawn C Lynch \\ Marjorie J Rah \\ Kimberly A Millard \\ Timothy W Morris}

Bausch \& Lomb Incorporated, Rochester, NY, USA
Correspondence: Marjorie J Rah

Bausch \& Lomb Incorporated, $1400 \mathrm{~N}$

Goodman St, Rochester, NY 14609, USA

Tel + I 585 4I3 6397

Email marjorie.rah@bausch.com
Purpose: To determine whether agents which are purportedly capable of inducing encystment of Acanthamoeba can recapitulate the signal when tested in differing formulations.

Methods: In accordance with the International Standard ISO 19045, Acanthamoeba castellanii ATCC 50370 trophozoites were cultured in antibiotic-free axenic medium, treated with test solutions, and encystment rates plus viability were measured via bright field and fluorescent microscopy. Test solutions included phosphate-buffered saline (PBS), borate-buffered saline, biguanide- and hydrogen peroxide $\left(\mathrm{H}_{2} \mathrm{O}_{2}\right)$-based biocides, propylene glycol (PG) and povidone (POV) ophthalmic demulcents, and one-step $\mathrm{H}_{2} \mathrm{O}_{2}$-based contact lens disinfection systems.

Results: Only PBS solutions with 0.25 ppm polyaminopropyl biguanide (PAPB) and increasing concentrations of PG and POV stimulated $A$. castellanii encystment in a dose-dependent manner, whereas PBS solutions containing $3 \% \mathrm{H}_{2} \mathrm{O}_{2}$ and increasing concentrations of PG and POV did not stimulate encystment. Borate-buffered saline and PBS/citrate solutions containing PG also did not stimulate encystment. In addition, no encystment was observed after 24 hours, 7 days, or 14 days of exposures of trophozoites to one-step $\mathrm{H}_{2} \mathrm{O}_{2}$ contact lens disinfection products or related solutions.

Conclusion: The lack of any encystment observed when trophozoites were treated with existing or new one-step $\mathrm{H}_{2} \mathrm{O}_{2}$ contact lens care products, as well as when trophozoites were exposed to various related test solutions, confirms that Acanthamoeba encystment is a complex process which depends upon simultaneous contributions of multiple factors including buffers, biocides, and demulcents.

Keywords: propylene glycol, contact lens care system, hydrogen peroxide disinfecting solution

\section{Introduction}

Acanthamoeba protozoa are ubiquitous pathogens found in both soil and aquatic environments, man-made structures and can also reside in healthy organisms. ${ }^{1,2}$ They are capable of causing a variety of human infections, including granulomatous amebic encephalitis in immune-compromised individuals, which affects the central nervous system and is often fatal. ${ }^{1}$ Acanthamoeba have been shown to serve as a reservoir for Legionella, with these bacteria persisting as endosymbionts within the amoebae, leading to the hypothesis that outbreaks of Legionnaire's disease may be directly related to presence of the Acanthamoeba. ${ }^{3}$ In healthy (ie, immune-competent) patients, Acanthamoeba spp. can also cause Acanthamoeba keratitis (AK), a painful infection of the cornea which can result in severe and permanent visual impairment, including blindness. ${ }^{4}$ The incidence of AK among contact lens wearers in the USA has historically been low, with estimates of one to two cases per million users. ${ }^{5}$ Approximately $80 \%-90 \%{ }^{6}$ of AK infections 
occur among contact lens wearers, however, with additional risk factors associated with noncompliance, such as improper storage and handling, as well as swimming or showering while wearing lenses. ${ }^{4}$ An increase in confirmed AK cases was observed at the beginning of 2004 and ultimately was linked to patients using the multipurpose contact lens cleaning solution Complete ${ }^{\circledR}$ MoisturePlus ${ }^{\mathrm{TM}}$ (CMP), resulting in its voluntary recall by Advanced Medical Optics in May $2007 .{ }^{7}$ The association of AK cases with CMP users was proposed to be due to stimulation of Acanthamoeba encystment observed following exposure to the CMP solution. ${ }^{8}$

Under conditions of environmental stress such as starvation and desiccation, Acanthamoeba trophozoites undergo transformation into a double-walled resistant cyst that is capable of remaining dormant, yet viable, until conditions favorable for growth return. This encystment process is a natural component of the Acanthamoeba life cycle, and cyst forms have been shown to resist disinfection by heat, chlorination, and treatment with multipurpose contact lens disinfecting solutions. ${ }^{9-16}$ Kilvington et al showed that over $20 \%$ of Acanthamoeba trophozoites exposed to CMP solution for 24 hours in lab experiments transformed into immature cysts. ${ }^{8}$ This study concluded that the demulcent propylene glycol (PG) was a causative factor in the CMP solution which promoted encystment, while importantly noting that additional factors, such as the buffering system of the solution, may have also been involved. ${ }^{8}$ The hypothesized influence of other (ie, non-PG) factors is supported by the fact that many currently available ophthalmic products (including a variety of other contact lens disinfecting solutions, contact lens packaging solutions, and lubricating eye drops) contain PG and to date have not been associated with Acanthamoeba encystment.

Ophthalmic demulcents are typically water-soluble polymers which may be topically applied to the eye to protect and lubricate mucous membrane surfaces as well as to relieve dryness and irritation. ${ }^{17}$ Ophthalmic demulcents are also present in various lens care disinfecting solutions to provide enhanced lubrication. PG is a liquid polyol monographed ophthalmic demulcent that acts as a water-binding moisturizer that can hold nearly three times its weight in water. ${ }^{18}$ Similarly, the demulcent povidone (POV) can retain water while also lubricating the surfaces to which it binds. Both are approved for human use as active ingredients in over-thecounter ophthalmic products at concentrations ranging from $0.2 \%$ to $1.0 \%$ for PG and $0.2 \%$ to $2.0 \%$ for POV. ${ }^{17}$

Previous studies have investigated the contribution of PG in multipurpose solutions on Acanthamoeba encystment.
Although one study concluded that PG was a primary factor in CMP solution-induced encystment, ${ }^{8}$ additional studies showed that solutions containing PG at similar concentrations did not stimulate encystment. ${ }^{19}$ Additionally, Acanthamoeba encystment has been shown to be influenced by different buffering agents. ${ }^{20}$ Furthermore, the impact of $\mathrm{PG}$ in $\mathrm{H}_{2} \mathrm{O}_{2}$ based disinfecting solutions has not been thoroughly studied. Given the previous studies showing PG as a causative factor of Acanthamoeba encystment plus the lack of similar testing for $\mathrm{H}_{2} \mathrm{O}_{2}$-based solutions, ${ }^{8}$ a series of model disinfecting solutions and commercially available lens care products were tested to directly assess the individual and combined impacts of different ophthalmic demulcents, chemical disinfectants, and buffering agents on Acanthamoeba encystment. The purpose of the study reported here was to determine whether agents which are purportedly capable of inducing encystment of Acanthamoeba can recapitulate the signal when tested in differing formulations. Such data have important implications for the development of contact lens disinfecting solutions concerning the types and concentrations of buffering agents and biocidal components to be used when considering Acanthamoeba encystment.

\section{Materials and methods Cultures and media}

A. castellanii ATCC 50370 (American Type Culture Collection, Manassas, Virginia, USA) was thawed from cryopreserved ampoules and grown for $3-4$ days at $28^{\circ} \mathrm{C} \pm 2^{\circ} \mathrm{C}$ in semi-defined axenic AC\#6 growth media lacking antibiotics. One day prior to testing, cells were passaged to fresh medium and incubated for an additional $20-24$ hours at $28^{\circ} \mathrm{C} \pm 2^{\circ} \mathrm{C}$. Following outgrowth, trophozoites were dislodged, washed three times in quarter-strength Ringer's solution (Oxoid BR0052G; Thermo Fisher Scientific, Waltham, MA, USA), and centrifuged at $500 \times g$ for 5 minutes at room temperature. Cells were counted using a disposable hemocytometer (C-chip Fuchs-Rosenthal DHC-F01, INCYTO; Drive Covington, GA, USA) and adjusted to $1.0 \times 10^{7}$ trophozoites $/ \mathrm{mL}$ immediately before testing. Neutralization buffer for $\mathrm{H}_{2} \mathrm{O}_{2}$-containing solutions (AC\#6-C) consisted of AC\#6 media supplemented with $0.1 \%$ filter-sterilized bovine liver catalase (Sigma C40; Sigma-Aldrich Co, St Louis, MO, USA).

\section{Test solutions}

Table 1 provides a complete listing along with key composition details for each test solution. A positive encystment control solution was adapted from both our own studies and previous reported studies: phosphate buffered saline (PBS) 
Table I Summary of test solutions in this study

\begin{tabular}{|c|c|c|}
\hline Test solution & Key components & Experiment \\
\hline PBS-PAPB-PG+ & PBS, 0.25 ppm PAPB, 0.5\% PG & Figure I \\
\hline PBS-PAPB-PG- & PBS, 0.25 ppm PAPB & Figure I \\
\hline BBS-PAPB-PG+ & BBS, 0.25 ppm PAPB, 0.5\% PG & Figure I \\
\hline BBS-PAPB-PG- & BBS, 0.25 ppm PAPB & Figure I \\
\hline $\mathrm{H}_{2} \mathrm{O}_{2}$ range & PBS, $1 \% \mathrm{PG}, \mathrm{H}_{2} \mathrm{O}_{2}$ range & Figure 2 \\
\hline PeroxiClear (PER-I), Bausch \& Lomb & PBS/citrate, I\% PG, $3 \% \mathrm{H}_{2} \mathrm{O}_{2}$ & Figure 3 \\
\hline PER-Ia & PER-I, minus PG & Figure 3 \\
\hline PER-Ib & PER-I, minus $\mathrm{H}_{2} \mathrm{O}_{2}$ & Figure 3 \\
\hline PER-IC & PER-I, minus $P G$ and $\mathrm{H}_{2} \mathrm{O}_{2}$ & Figure 3 \\
\hline PG range in PAPB & PBS, 0.25 ppm PAPB, $0 \%-1 \%$ PG & Figure 4 \\
\hline $\mathrm{POV}$ range in $\mathrm{PAPB}$ & PBS, 0.25 ppm PAPB, $0 \%-2 \%$ POV & Figure 4 \\
\hline PG range in $\mathrm{H}_{2} \mathrm{O}_{2}$ & PBS, 0.25 ppm $3 \% \mathrm{H}_{2} \mathrm{O}_{2}, 0 \%-1 \%$ PG & Figure 4 \\
\hline $\mathrm{POV}$ range in $\mathrm{H}_{2} \mathrm{O}_{2}$ & PBS, 0.25 ppm $3 \% \mathrm{H}_{2} \mathrm{O}_{2}, 0 \%-2 \%$ POV & Figure 4 \\
\hline PER-I & PBS/citrate, I\% PG, $3 \% \mathrm{H}_{2} \mathrm{O}_{2}$ & Figure 5 \\
\hline Clear Care (PER-2), Alcon Laboratories & PBS, $3 \% \mathrm{H}_{2} \mathrm{O}_{2}$ & Figure 5 \\
\hline
\end{tabular}

Abbreviations: BBS, borate-buffered saline; BBS-PAPB-PG+, solution containing BBS, PAPB and PG; BBS-PAPB-PG-, solution containing BBS and PAPB, without PG; PAPB, polyaminopropyl biguanide; PBS, phosphate-buffered saline; PBS-PAPB-PG+, solution containing PBS, PAPB and PG; PBS-PAPB-PG-, solution containing PBS and PAPB, without PG; PER-Ia, PeroxiClear solution lacking propylene glycol; PER-Ib, PeroxiClear solution lacking $\mathrm{H}_{2} \mathrm{O}_{2}$; PER-Ic, PeroxiClear solution lacking propylene glycol and $\mathrm{H}_{2} \mathrm{O}_{2}$; PG, propylene glycol; POV, povidone.

solution containing polyaminopropyl biguanide (PAPB, also referred to as polyhexamethylene biguanide) and PG (PBS-PAPB-PG+). ${ }^{21}$ The non-buffer components of PBSPAPB-PG+ were also formulated as a borate-buffered saline (BBS) solution containing PAPB and PG (BBS-PAPB-PG+) to investigate the role of different buffering agents. Two different ophthalmic demulcents, PG and POV, were added at their respective US Food and Drug Administration (FDA) monographed ranges ${ }^{17}$ into either PAPB or $\mathrm{H}_{2} \mathrm{O}_{2}$-containing solutions. A series of $\mathrm{H}_{2} \mathrm{O}_{2}$ concentrations $(0 \%-3 \%)$ was also tested in the presence of $1 \%$ PG. A new one-step $\mathrm{H}_{2} \mathrm{O}_{2}$-based contact lens care product, PeroxiClear (PER-1; Bausch \& Lomb, Rochester, NY, USA) was tested in parallel with another $\mathrm{H}_{2} \mathrm{O}_{2}$-based lens care product, Clear Care (PER-2; Alcon Laboratories, Fort Worth, TX, USA). Derivatives of the PER-1 solution which lacked PG, $\mathrm{H}_{2} \mathrm{O}_{2}$, or both (PER-1a, PER-1b, PER-1c, respectively) were also tested. Neutralization of $\mathrm{H}_{2} \mathrm{O}_{2}$-containing solutions was confirmed by measuring residual $\mathrm{H}_{2} \mathrm{O}_{2}$ levels using a Mettler Toledo T50 Autotitrator with a modified 4-hour method adapted from USP 38 monograph. The monograph describes a titrimetric system for assaying the concentration of hydrogen peroxide in a topical solution. The titrant used was $0.1 \mathrm{~N}$ potassium permanganate with the endpoint detected using Mettler Toledo LabX light titration, version 3.1.1.0. Each milliliter of the titrant used was equivalent to $1.701 \mathrm{mg}$ of hydrogen peroxide. A modification of the sample size along with programming the software to automatically dispense the titrant at a controlled rate allowed for the detection of hydrogen peroxide at levels between 0 and 30,000 ppm.

\section{Encystment testing}

Encystment rates for each solution were measured as described in International Standard ISO 19045:2015: Ophthalmic optics - contact lens care products - method for evaluating Acanthamoeba encystment by contact lens care products" (hereafter, "ISO 19045"), ${ }^{22}$ with modifications as needed to accommodate testing with one-step $\mathrm{H}_{2} \mathrm{O}_{2}$ disinfecting systems. Assays using solutions containing $\mathrm{H}_{2} \mathrm{O}_{2}$ were performed in manufacturer-provided neutralizing lens cases containing platinum discs which catalyze the conversion of $\mathrm{H}_{2} \mathrm{O}_{2}$ to $\mathrm{H}_{2} \mathrm{O}$ and $\mathrm{O}_{2}$. Lens cases were filled with $10 \mathrm{~mL}$ of test solution and inoculated to give a final concentration of $1.0 \times 10^{5}$ trophozoites $/ \mathrm{mL}$, then immediately capped and incubated at room temperature $\left(20^{\circ} \mathrm{C} \pm 2^{\circ} \mathrm{C}\right)$. At specified time points, the detergent sarkosyl (Sigma 61747; Sigma-Aldrich Co) was added to lens cases to lyse any remaining trophozoites, while the fluorescent stain calcofluor-white (Sigma F3543; Sigma-Aldrich Co), which binds to cellulose within cyst walls, ${ }^{23}$ was used to identify cysts. Exposure to the sarkosyl/calcofluor-white mixture $(150 \mathrm{mg} / 10 \mathrm{mg}$ per $1.0 \mathrm{~mL}$ in purified water) did not exceed 5 minutes to ensure that only trophozoites, and not cysts, would be lysed. Test solutions which lacked $\mathrm{H}_{2} \mathrm{O}_{2}$ were tested in multi-well plates containing $3 \mathrm{~mL}$ of test solution and inoculated to give a final concentration of $1.0 \times 10^{5}$ trophozoites $/ \mathrm{mL}$, and all other steps were performed as described earlier. Samples were enumerated using an Olympus BX51 upright epifluorescence microscope by comparing manual counts under bright field illumination to epifluorescence (excitation $350 \mathrm{~nm} / \mathrm{emission} 460 \mathrm{~nm}$ ). Rounded cellular bodies with bright white fluorescence 
were counted as cysts. Encystment was expressed as a percentage of cysts observed after solution exposure relative to inoculum control counts, corrected for background cyst count obtained at 0 hour. Final percentages were averaged from three independent assays. For encystment studies that were carried out to 14 days, viable amoebae were also determined as described later in this work.

\section{Viability counts}

To enumerate surviving amoebae after 14 days of incubation with test solutions, an agar overlay method ${ }^{24}$ was used, with modifications. Briefly, $1 \mathrm{~mL}$ samples were removed from lens cases after 14 days' incubation and neutralized in AC\#6-C for 10 minutes. Samples were again serially diluted (1:10) in AC\#6-C, then pelleted by centrifugation at $1,000 \times g$ for 5 minutes and resuspended into $100 \mu \mathrm{L}$ of neutralizing buffer. To this, $100 \mu \mathrm{L}$ of Escherichia coli (ATCC 33876, 5.0×10\%/ $\mathrm{mL}$ ) and $3 \mathrm{~mL}$ of molten top agar (10.0 $\mathrm{g}$ tryptone; $5.0 \mathrm{~g} \mathrm{NaCl}$; $7.0 \mathrm{~g} \mathrm{Bacto}^{\mathrm{TM}}$ agar (BD Biosciences 214010; BD Biosciences, San Jose, CA, USA) held at $45^{\circ} \mathrm{C}$ were added and vortexed to mix thoroughly. The top agar mixture was then poured onto solidified $\mathrm{SM} / 5$ agar plates (2.0 g proteose peptone, 2.0 $\mathrm{g}$ yeast extract, $2.0 \mathrm{~g}$ glucose, $2.0 \mathrm{~g} \mathrm{MgSO}_{4}$, and $15.0 \mathrm{~g}$ Bacto agar in $1.0 \mathrm{~L}$ of purified $\mathrm{H}_{2} \mathrm{O}$ ) and incubated at $28^{\circ} \mathrm{C} \pm 2{ }^{\circ} \mathrm{C}$ for 5 days. Viable amoebae were enumerated by counting visible plaques formed against a background of $E$. coli growth within the top agar, and recorded as plaque-forming units.

\section{Stand-alone biocidal efficacy}

Testing of one-step $\mathrm{H}_{2} \mathrm{O}_{2}$ disinfecting solutions was adapted from previously described methods. ${ }^{11,19}$ Independent duplicate assays were performed in new lens cases containing neutralizing platinum-coated discs. Lens cases were filled with $10 \mathrm{~mL}$ of either PER-1 or PER-2 and inoculated to give a final concentration of $5.0 \times 10^{4}$ trophozoites $/ \mathrm{mL}$, then immediately capped. At each time point, samples were neutralized with AC\#6-C for 10 minutes, then serially diluted (1:10) in tubes containing AC\#6-C. Aliquots from the dilutions were added to the wells of a 96 -well plate and incubated at $28^{\circ} \mathrm{C} \pm 2^{\circ} \mathrm{C}$. Wells were then microscopically inspected over a 14-day outgrowth period for evidence of trophozoite growth and replication. $\log _{10}$ reduction values were averaged from independent duplicate assays as determined by the most-probable number method using Spearman-Karber computations. ${ }^{25}$

\section{Statistical methods}

All analyses were run using Minitab ${ }^{\circledR}$ 17.2.1 Statistical Software (Minitab Inc., State College, PA, USA); Bonett's method was used to determine any statistical differences in variances between two-sample sets using an alpha $=0.05$. Any statistical differences in the variances were accounted for in the comparison of the mean values. Two-sample $t$-tests were used for comparing the means between the control and test sample sets at $95 \%$ confidence interval. If the $P$-value was less than or equal to 0.05 , the means were considered to be significantly different between the two-sample sets.

\section{Results}

Across multiple experiments, the positive encystment control solution PBS-PAPB-PG+ (containing 0.5\% PG and 0.25 ppm PAPB in a phosphate-buffered system) consistently yielded encystment rates of $\geq 50 \%$ (Figures 1 and 2 ). To assess the influence of different buffering systems on $A$. castellanii encystment, the non-buffer system components of PBSPAPB-PG+ were reformulated into a borate-buffered system BBS-PAPB-PG+. After incubation with BBS-PAPB-PG+ for 24 hours, A. castellanii trophozoites did not encyst (Figure 1). The mean encystment rate of the control solution PBS-PAPB-PG+ was significantly higher than each of the test solutions (solution containing PBS and PAPB, without PG [PBS-PAPB-PG-], BBS-PAPB-PG+, and solution containing BBS and PAPB without PG [BBS-PAPB-PG-] $(P<0.05)$.

Next, a series of PBS/citrate-buffered solutions containing a concentration of $1.0 \% \mathrm{PG}$ and a range of $\mathrm{H}_{2} \mathrm{O}_{2}$ concentrations from $0 \%$ to $3 \%$ were tested, none of which induced encystment of $A$. castellanii (Figure 2). The mean encystment rate of the positive control (PBS-PAPB-PG+) was

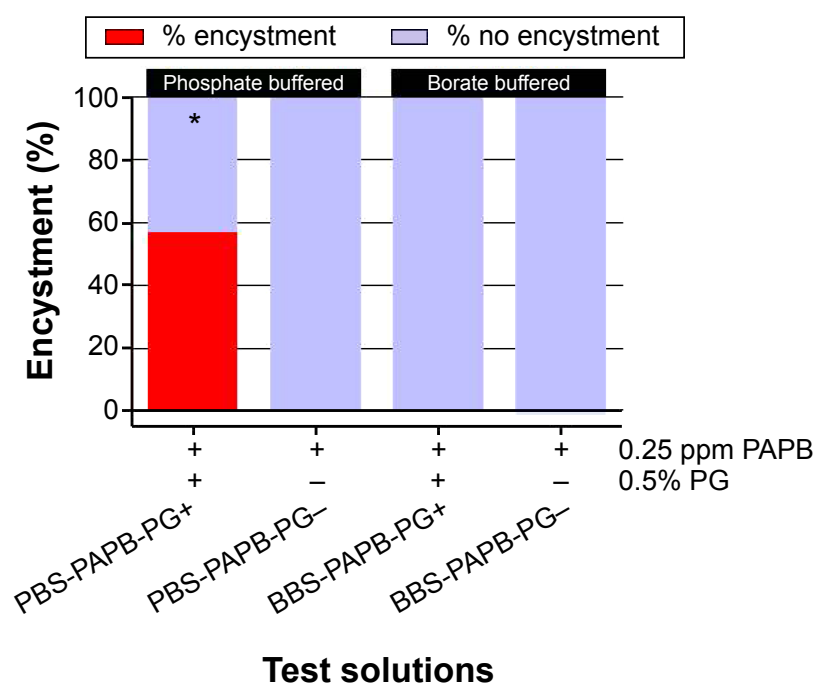

Figure I Impact of buffer agents on Acanthamoeba castellanii encystment rates. Note: Red bar represents percentage of trophozoites that encysted after 24 hours' incubation, while blue bars represent percentage of population that did not $(* P<0.05)$. Abbreviations: BBS, borate-buffered saline; BBS-PAPB-PG+, solution containing BBS, PAPB and PG; BBS-PAPB-PG-, solution containing BBS and PAPB, without PG; PAPB, polyaminopropyl biguanide; PBS, phosphate-buffered saline; PBS-PAPB-PG+, solution containing PBS, PAPB and PG; PBS-PAPB-PG-, solution containing PBS and PAPB, without PG; PG, propylene glycol; POS, positive. 


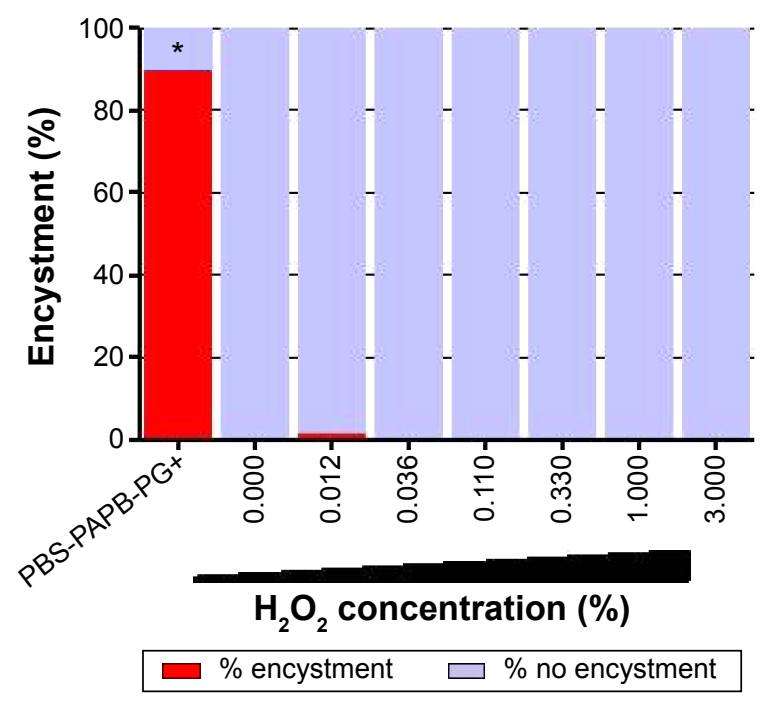

Figure 2 Encystment rates of Acanthamoeba castellanii exposed to PBS-PAPB-PG+ control and to I\% PG with varying concentrations of $\mathrm{H}_{2} \mathrm{O}_{2}$.

Note: Red bar represents percentage of trophozoites that encysted after 24 hours incubation, while blue bars represent percentage of population that $\operatorname{did}$ not $(* P<0.05)$. Abbreviations: PAPB, polyaminopropyl biguanide; PBS, phosphate-buffered saline; PBS-PAPB-PG+, solution containing PBS, PAPB and PG; PG, propylene glycol.

significantly higher than all varying concentrations of $\mathrm{H}_{2} \mathrm{O}_{2}$ $(P<0.05)$, while among the varying concentrations of $\mathrm{H}_{2} \mathrm{O}_{2}$, no significant differences were observed $(P>0.05)$.

To assess $A$. castellanii encystment with regards to the addition of ophthalmic demulcents to model disinfecting solutions, phosphate-buffered solutions that contained either 0.25 ppm PAPB or $3 \% \mathrm{H}_{2} \mathrm{O}_{2}$ were prepared. Both POV and PG were added to each at dose levels spanning their respective FDA monograph concentration ranges (POV: 0.0\%-2.0\%, PG: 0.0\%-1.0\%; monograph ophthalmic demulcent concentration ranges defined by the FDA Code of Federal Regulations for use in over-the-counter ophthalmic drug products). ${ }^{17}$ After 24 hours' incubation, encystment rates for solutions containing 0.25 ppm PAPB increased in a dose-dependent manner for both PG and POV (88\% and 14\%, respectively, at their highest test concentration). For both PAPB and $\mathrm{H}_{2} \mathrm{O}_{2}$ test solutions, the mean encystment rates for different concentrations of POV and PG were compared to their respective base solution (ie, $0.0 \%$ POV or PG) (Figure 3). For the PAPB test solutions containing POV, only the formulation of $2.0 \%$ POV was significantly greater than the solution containing no $(0.0 \%)$ POV $(P<0.05)$. Mean encystment rates of PAPB test solutions containing $\mathrm{PG}$ were significantly greater than that of the PAPB solution without PG $(0.0 \%)$ for all concentrations tested $(P<0.05)$. Solutions containing 3\% $\mathrm{H}_{2} \mathrm{O}_{2}$ did not induce encystment (ie, $\leq 1 \%$ ) regardless of the presence or absence of demulcents. For all concentrations of POV with the $\mathrm{H}_{2} \mathrm{O}_{2}$ test solutions, the mean encystment rates were not significantly different from the solution

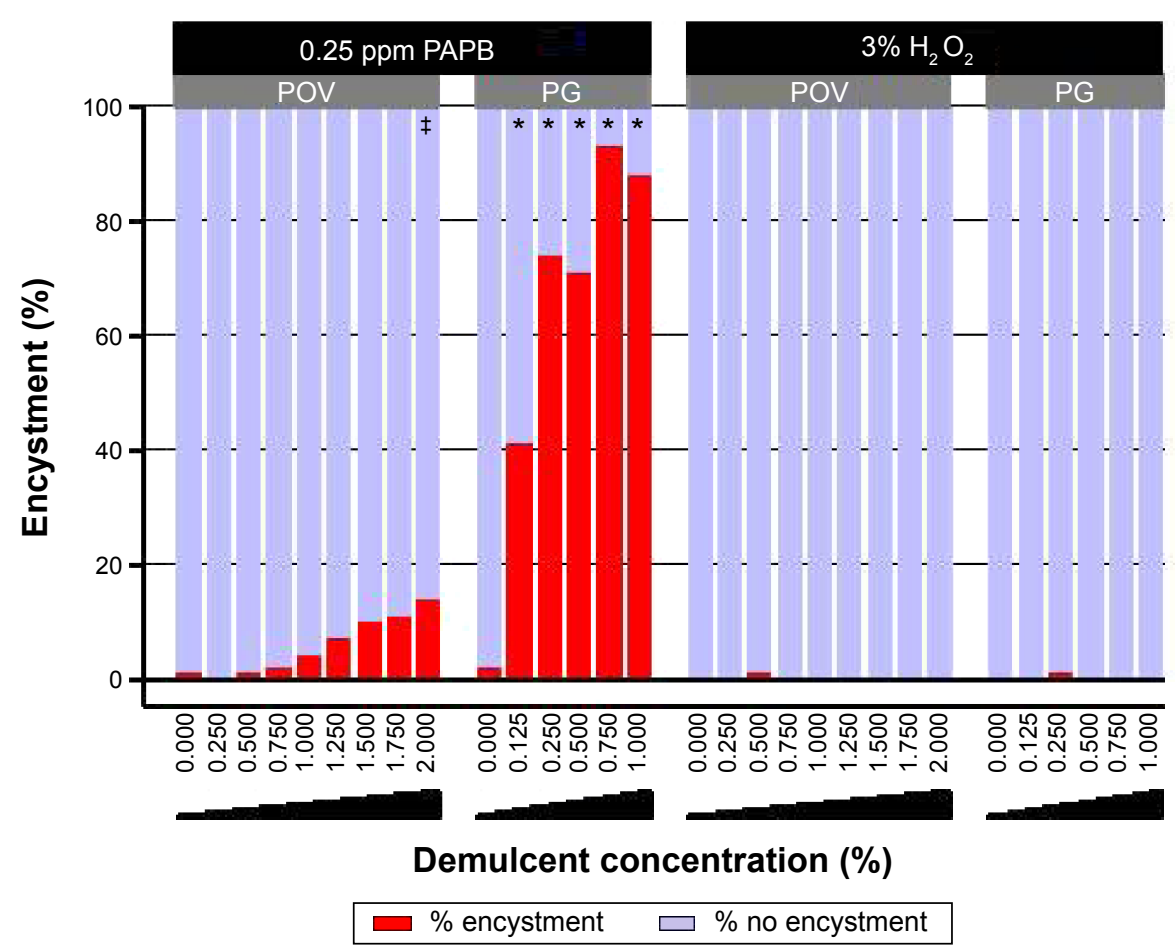

Figure 3 Encystment rates of Acanthamoeba castellanii exposed to phosphate-buffered solutions that contained either 0.25 ppm polyaminopropyl biguanide (PAPB) or $3 \%$ $\mathrm{H}_{2} \mathrm{O}_{2}$ with increasing concentrations of povidone (POV) and propylene glycol (PG).

Notes: Red bars represent percentage of trophozoites that encysted after 24 hours’ incubation, while blue bars represent percentage of population that did not ( $¥ P<0.05$ for PAPB-2.0\% POV vs PAPB- $0.0 \%$ POV; $* P<0.05$ for all PAPB-PG containing solutions vs PAPB- $0.0 \%$ PG). 


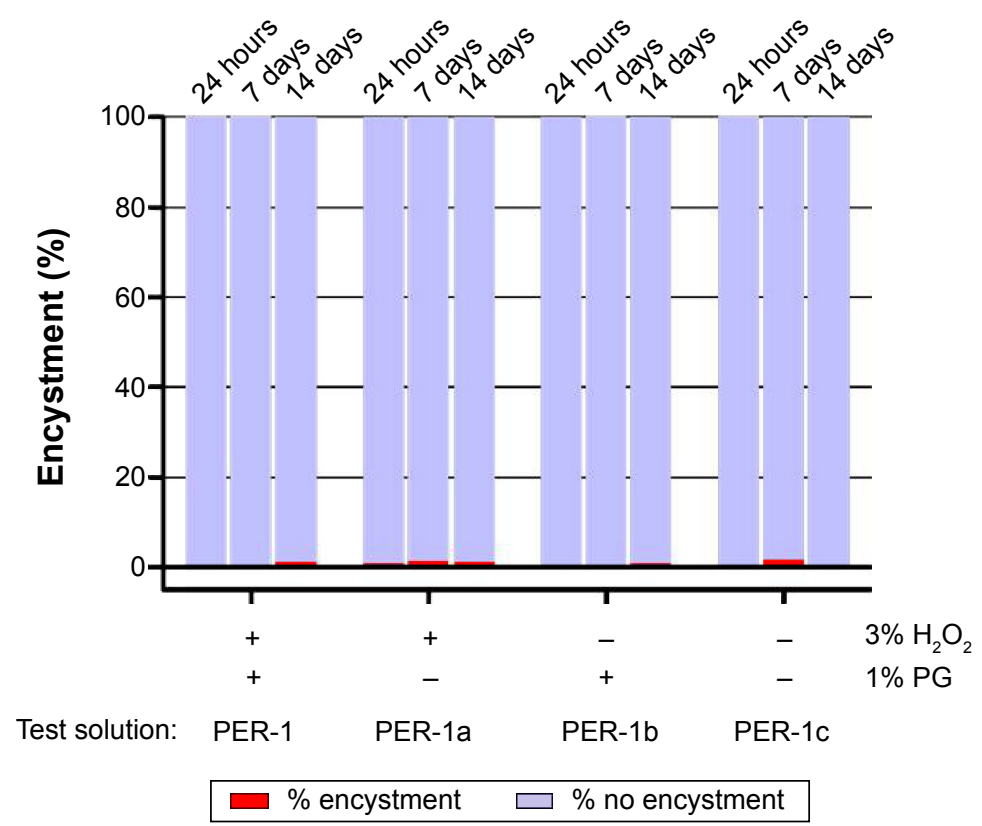

Figure 4 Encystment rates of Acanthamoeba castellanii trophozoites exposed to PeroxiClear (PER-I), PeroxiClear solution lacking propylene glycol (PER-Ia), PeroxiClear solution lacking $\mathrm{H}_{2} \mathrm{O}_{2}$ (PER-Ib), and PeroxiClear solution lacking propylene glycol and $\mathrm{H}_{2} \mathrm{O}_{2}$ (PER-Ic) for 24 hours, 7 days, and 14 days. No significant differences were observed across any of the solutions tested regardless of the time point $(P>0.05)$.

Notes: Red bars represent percentage of trophozoites that encysted, while blue bars represent percentage of population that did not. The + and - symbols indicate whether or not the $3 \% \mathrm{H}_{2} \mathrm{O}_{2}$ and/or the $1 \%$ PG were included within the solution.

Abbreviation: $\mathrm{PG}$, propylene glycol.

containing no POV $(P>0.05)$. Similarly, for the $\mathrm{H}_{2} \mathrm{O}_{2}$ test solutions containing PG, the mean encystment rate of the test solution containing a $0.0 \%$ concentration of PG was not significantly different than the test solution with increasing concentrations of PG $(P>0.05)$.

Label instructions for the new one-step $\mathrm{H}_{2} \mathrm{O}_{2}$ disinfecting solution (PER-1) tested included simultaneous disinfection and neutralization with a minimum of a 4-hour soak time followed by storage of disinfected contact lenses for up to 7 days. Considering these storage instructions, the potential for encystment during a simulated long-term storage condition was tested as follows. When $A$. castellanii was added to PER-1 and its derivatives, PER-1a, PER-1b, and PER-1c (Table 1), and incubated for 24 hours, 7 days, or 14 days, encystment rates for all solutions at each time point were $\leq 2 \%$, which was significantly less $(P<0.05)$ than the positive control (Figure 4$)$. Surviving cells from inoculated lens cases stored for 14 days were also enumerated with no recovery of Acanthamoeba observed for PER-1 and PER-1a solutions (both of which contained $3 \% \mathrm{H}_{2} \mathrm{O}_{2}$ ), whereas 2,200 and 1,100 amoeba/mL, respectively, were recovered from PER-1b and PER-1c (both of which did not contain $\mathrm{H}_{2} \mathrm{O}_{2}$ ).

Finally, both of the one-step $\mathrm{H}_{2} \mathrm{O}_{2}$ lens care products PER-1 and PER-2 - were evaluated for encystment effects as well as for stand-alone biocidal efficacy against $A$. castellanii trophozoites. Encystment rates for both products were $<1 \%$ after 24 hours' incubation, significantly $(P<0.05)$ lower than the positive control solution (Figure 5A). Average $\log _{10}$ reduction results at 4,6 , and 24 hours were $4.3,4.3$, and 4.3, respectively, for PER-1, and 4.1, 4.2, and 4.1, respectively, for PER-2 (Figure 5B).

\section{Discussion}

While standardized performance criteria are lacking for biocidal efficacy and encystment testing of Acanthamoeba, better understanding of the factors that contribute to the organism's ability to evade treatment are needed for the development of improved contact lens care systems. The study presented here demonstrated that stimulation of $A$. castellanii encystment by model contact lens disinfecting solutions can be dependent upon multifactorial contributions from various solution components. Among the known factors, the buffering system and biocidal agents used in a solution were shown to directly influence encystment rates under some (but not all) test conditions. In addition, exposure of $A$. castellanii trophozoites to a variety of test solutions which contained PG (including a new $\mathrm{H}_{2} \mathrm{O}_{2}$-based contact lens disinfecting solution) did not stimulate encystment.

Previous studies have demonstrated that multipurpose solutions containing PG may stimulate Acanthamoeba 

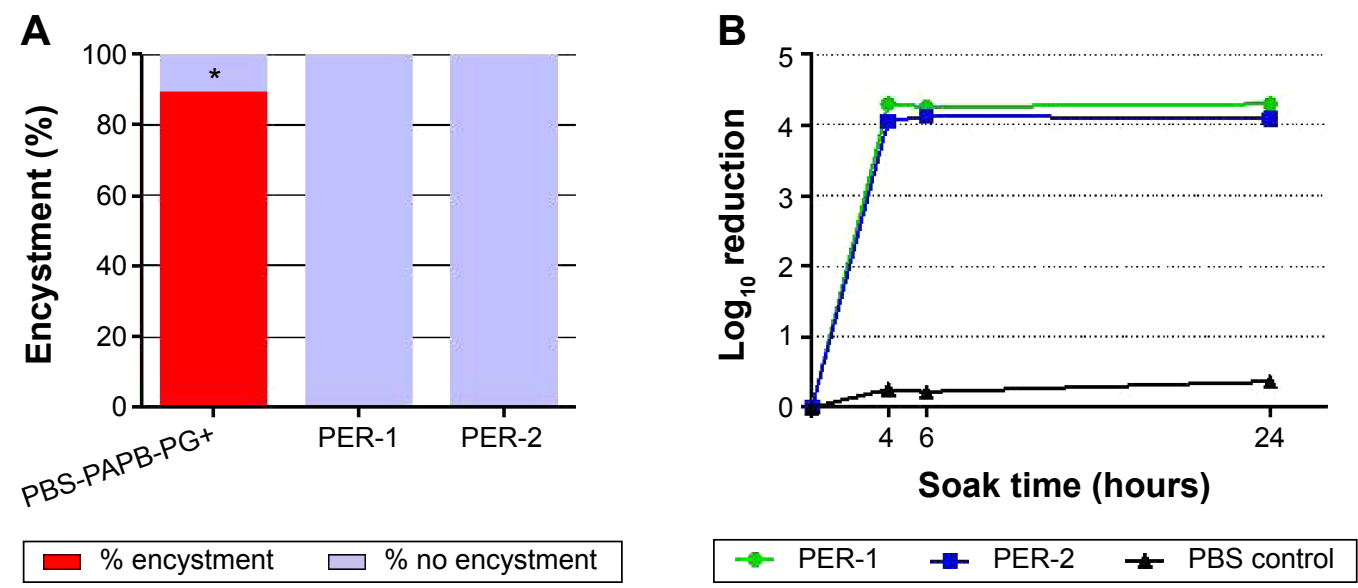

Figure 5 (A) Encystment rates of Acanthamoeba castellanii exposed to PBS-PAPB-PG+ control, PeroxiClear (PER-1), and Clear Care ${ }^{\circledR}$ (PER-2) solutions. Encystment rates for both PER-I and PER-2 were $<1 \%$ after 24 hours' incubation, significantly $(* P<0.05)$ lower than the positive control solution. (B) For stand-alone biocidal efficacy testing, $\log _{10}$ reduction values of $A$. castellanii exposed to PER-I, and PER-2 solutions reached complete kill at 6 and 24 hours, while PBS control did not.

Note: Red bar represents percentage of trophozoites that encysted after 24 hours' incubation, while blue bars represent percentage of population that did not. Abbreviations: PAPB, polyaminopropyl biguanide; PBS, phosphate-buffered saline; PBS-PAPB-PG+, solution containing PBS, PAPB and PG.

encystment to varying degrees. In one study, CMP solution was reported to induce encystment at a rate of $20.8 \%$ in A. castellanii, while Menicare Soft and Epica Cold (both also containing PG) yielded only $1.2 \%$ and $2.4 \%$ cysts, respectively. ${ }^{8}$ Additionally, when several multipurpose solutions were supplemented with $0.5 \%$ PG, encystment rates were unaffected for some solutions, while for others, encystment rates slightly increased. Yet, in all instances, the rates remained much lower than those reported for the CMP solution. ${ }^{8}$ It has been suggested that these increases in encystment rates were due to increased osmolality resulting from the addition of PG to the solutions. ${ }^{19}$ Later studies by Lonnen et al confirmed the low levels of encystment previously observed in Menicare Soft and Epica Cold. ${ }^{19}$ However, a multipurpose solution which lacked PG was also shown to induce Acanthamoeba encystment. The authors hypothesized that this encystment in the absence of PG was potentially due to the phosphate buffer component of that test solution. ${ }^{19}$

A number of factors have been proposed to contribute to Acanthamoeba encystment associated with a solution, including osmolality, ${ }^{19,26}$ intrinsic biocidal activity, ${ }^{27}$ and buffering agents. ${ }^{8,19}$ Indeed, various multipurpose solutions that contain PG either do not induce encystment or result in much lower encystment rates than those observed with CMP solution. ${ }^{8}$ The phosphate-buffered positive control solution (PBS-PAPB-PG+) used in the present study, which contained PG, was compared to a model solution composed of the same ingredients at identical concentrations in a borate-buffered positive control solution - BBS-PAPB-PG+. Encystment was not observed after 24 hours' incubation with BBS-PAPB-PG+, suggesting that the encystment signal observed in PBS-PAPB-PG+ can be attributed to the combined effects of the phosphate buffer and PG. In addition, the PER-1c solution (containing 1.0\% PG in a PBS/citrate-buffered formulation without a biocidal agent) also did not induce encystment, even with prolonged incubation up to 14 days (Figure 5). Further, studies have also shown that Acanthamoeba culbertsoni encystment was inhibited by citrate buffer, while Tris buffer lysed trophozoites. ${ }^{20}$ Taken together, these results clearly demonstrate that encystment of Acanthamoeba spp. is mediated by the overall composition of a solution, including its buffering system in conjunction with other components.

PG is classified by the FDA as a liquid polyol demulcent, safe for use in over-the-counter products at concentrations ranging from $0.2 \%$ to $1.0 \%{ }^{17}$ Its primary function in contact lens disinfecting solutions is to provide enhanced lubrication and wettability of contact lenses. POV, another demulcent often present in eye drop formulations, is similarly approved for use at concentrations ranging from $0.2 \%$ to $2.0 \% .{ }^{17}$ While neither demulcent has been shown to confer toxicity within its monograph concentration range, demulcents such as PG are capable of raising the osmolality of a solution. Indeed, hyperosmolality has been shown to cause encystment in pathogenic strains of Acanthamoeba. ${ }^{28}$ This was taken into consideration in the present study by controlling for osmolality and adjusting all test solutions to a specified osmolality target of 290-300 mOsm/ $\mathrm{kg}$, within the physiologically biocompatible range found in most commercially available contact lens care solutions. Here, the PG and POV demulcents showed a dose-dependent stimulation of encystment only when present in PBS-based solutions which also contained $0.25 \mathrm{ppm}$ PAPB. When the same concentrations 
of PG and POV were instead present in PBS solutions containing $3 \% \mathrm{H}_{2} \mathrm{O}_{2}$, neither demulcent stimulated encystment. While the intrinsic biocidal activity of $\mathrm{H}_{2} \mathrm{O}_{2}$ is typically very strong against Acanthamoeba trophozoites, it is important to note that the $\mathrm{H}_{2} \mathrm{O}_{2}$ experiments were carried out in neutralizing lens cases, where after 4 hours of incubation, the $\mathrm{H}_{2} \mathrm{O}_{2}$ levels were $<100 \mathrm{ppm}$ (data not shown). For encystment testing in this study, all solution exposures were for at least 24 hours, so unless complete kill had occurred during the initial 4 hours, there would still have been viable amoebae present and exposed to the PBS containing PG and POV for the remainder of the assay. Moreover, when the concentration of $\mathrm{PG}$ was held at a constant $1 \%$ and the concentration of $\mathrm{H}_{2} \mathrm{O}_{2}$ was dosed from $0 \%$ to $3 \%$, again no encystment was detected for any concentration of $\mathrm{H}_{2} \mathrm{O}_{2}$ tested. This is particularly relevant for $\mathrm{H}_{2} \mathrm{O}_{2}$ concentrations substantially below $3 \%$ where biocidal activity is expected to be reduced. Specifically, if encystment stimulation by PG is dependent on a sublethal insult, it is possible that exposure to low (ie, less effective) levels of $\mathrm{H}_{2} \mathrm{O}_{2}$ could result in stimulation of encystment. However, this was not observed, thus further supporting the hypothesis that encystment is dependent on more than just individual components of a solution.

Labeling instructions for the $\mathrm{H}_{2} \mathrm{O}_{2}$-based products PER-1 and PER-2 indicate that contact lenses may be stored for up to 7 days following the initial 4-hour (PER-1) or 6-hour (PER-2) disinfection period. Therefore, we also examined the influence of prolonged storage up to 14 days on Acanthamoeba encystment using a variety of formulations with and without both $3 \% \mathrm{H}_{2} \mathrm{O}_{2}$ and $1.0 \%$ PG components. No encystment was observed for any of these solutions after 24 hours', 7 days', or 14 days' storage in $\mathrm{H}_{2} \mathrm{O}_{2}$-neutralizing lens cases (Figure 4). This is particularly notable given that one of these solutions lacked any $\mathrm{H}_{2} \mathrm{O}_{2}$ while still containing $1.0 \%$ PG. Similarly, no encystment was detected with other commercially available one-step $\mathrm{H}_{2} \mathrm{O}_{2}$ solutions also incubated for 24 hours, 7 days, and 14 days (data not shown). Enumeration of surviving Acanthamoeba after 14 days' incubation in the lens case demonstrated that, as expected, the $\mathrm{H}_{2} \mathrm{O}_{2}$-containing solutions were highly effective (ie, near complete kill), while in contrast, substantial numbers of viable amoebae were recovered from solutions that lacked $\mathrm{H}_{2} \mathrm{O}_{2}$. Given that prolonged starvation is commonly used to create mature cysts in the laboratory, the recovery of viable amoebae with no evidence of encystment after 14 days is somewhat unexpected and warrants future investigation.

The ability of pathogenic Acanthamoeba to undergo encystment presents a particular challenge to lens care disinfecting systems because the cyst forms survive longer and are less susceptible to a variety of antimicrobial treatments. ${ }^{9,11-15}$ While the contribution (if any) of cyst forms to initiating AK infections remains unclear, a contact lens disinfecting solution that enhances encystment could potentially be problematic. Recent reports have suggested pseudocyst formation as an alternative stress response to encystment. ${ }^{29}$ Of note, pseudocyst formation was suggested to be caused by $\mathrm{PG},{ }^{30}$ although the testing and detection methods differed from those reported here. Per the ISO 19045 encystment standard, ${ }^{22}$ the cysts observed in our studies were not characterized as either pseudocysts or immature cysts; however, this distinction merits further study. In particular, it would be valuable to better understand the onset of these two reported cyst forms in order to delineate what differences may influence their respective formation, as well as any risk factors associated with these forms for contact lens-associated AK infections. Changes of trophozoites observed in the present study were shown to be greatly influenced by both buffering and biocidal components of the challenge solutions and demonstrate the importance of a whole system, rather than individual components, on Acanthamoeba encystment.

\section{Conclusion}

Overall, the results reported here confirm that encystment associated with PG exposure is dependent on multiple factors, including buffering agents and biocidal components, and that these additional factors should be considered as a whole when investigating encystment effects. Further examination of the biocidal agents commonly used in contact lens disinfecting solutions and their interactions with other ingredients (including multicomponent buffering systems) would provide a more comprehensive understanding of Acanthamoeba encystment. This knowledge could facilitate the rational invention and development of new contact lens care solutions with improved safety and efficacy.

\section{Acknowledgement}

The authors would like to thank Sara Irizarry for her contributions to the manuscript.

\section{Disclosure}

All authors were employees of Bausch \& Lomb Incorporated at the time of this work. The authors report no other conflicts of interest in this work.

\section{References}

1. Marciano-Cabral F, Cabral G. Acanthamoeba spp. as agents of disease in humans. Clin Microbiol Rev. 2003;16(2):273-307.

2. Mergeryan $H$. The prevalence of Acanthamoeba in the human environment. Rev Infect Dis. 1991;13 Suppl 5:S390-S391. 
3. Cirillo JD, Falkow S, Tompkins LS. Growth of Legionella pneumophila in Acanthamoeba castellanii enhances invasion. Infect Immun. 1994; 62(8):3254-3261.

4. Centers for Disease Control and Prevention (CDC). Acanthamoeba keratitis FAQs. Atlanta, GA: CDC; nd [August 21, 2012]. Available from: http://www.cdc.gov/parasites/acanthamoeba/gen_info/acanthamoeba_keratitis.html. Accessed March 27, 2014.

5. Schaumberg DA, Snow KK, Dana MR. The epidemic of Acanthamoeba keratitis: where do we stand? Cornea. 1998;17(1):3-10.

6. Ross J, Roy SL, Mathers WD, et al. Clinical characteristics of Acanthamoeba keratitis infections in 28 states, 2008 to 2011. Cornea. 2014; 33(2):161-168.

7. Verani JR, Lorick SA, Yoder JS, et al; Acanthamoeba Keratitis Investigation Team. National outbreak of Acanthamoeba keratitis associated with use of a contact lens solution, United States. Emerg Infect Dis. 2009; 15(8):1236-1242.

8. Kilvington S, Heaselgrave W, Lally JM, Ambrus K, Powell H. Encystment of Acanthamoeba during incubation in multipurpose contact lens disinfectant solutions and experimental formulations. Eye Contact Lens 2008;34(3):133-139.

9. Borazjani RN, Kilvington S. Efficacy of multipurpose solutions against Acanthamoeba species. Cont Lens Anterior Eye. 2005;28(4): 169-175.

10. Coulon C, Collignon A, McDonnell G, Thomas V. Resistance of Acanthamoeba cysts to disinfection treatments used in health care settings. J Clin Microbiol. 2010;48(8):2689-2697.

11. Hughes R, Kilvington S. Comparison of hydrogen peroxide contact lens disinfection systems and solutions against Acanthamoeba polyphaga Antimicrob Agents Chemother. 2001;45(7):2038-2043.

12. Khunkitti W, Lloyd D, Furr JR, Russell AD. Acanthamoeba castellanii growth, encystment, excystment and biocide susceptibility. $J$ Infect. 1998;36(1):43-48.

13. Kilvington S, Lam A. Development of standardized methods for assessing biocidal efficacy of contact lens care solutions against Acanthamoeba trophozoites and cysts. Invest Ophthalmol Vis Sci. 2013;54(7): $4527-4537$.

14. Kobayashi T, Gibbon L, Mito T, Shiraishi A, Uno T, Ohashi Y. Efficacy of commercial soft contact lens disinfectant solutions against Acanthamoeba. Jpn J Ophthalmol. 2011;55(5):547-557.

15. Mowrey-McKee M, George M. Contact lens solution efficacy against Acanthamoeba castellani. Eye Contact Lens. 2007;33(5):211-215.

16. Storey MV, Winiecka-Krusnell J, Ashbolt NJ, Stenström TA. The efficacy of heat and chlorine treatment against thermotolerant Acanthamoebae and Legionellae. Scand J Infect Dis. 2004;36(9): 656-662.

17. CRF - Code of Federal Regulations Title 21, Vol 5, Sec 349.12. Revised April 1, 2013. US Food and Drug Administration; US Department of Health \& Human Services. Available from: http:/www.accessdata. fda.gov/scripts/cdrh/cfdocs/cfcfr/CFRSearch.cfm. Accessed March 27, 2014.

Clinical Ophthalmology

\section{Publish your work in this journal}

Clinical Ophthalmology is an international, peer-reviewed journal covering all subspecialties within ophthalmology. Key topics include: Optometry; Visual science; Pharmacology and drug therapy in eye diseases; Basic Sciences; Primary and Secondary eye care; Patient Safety and Quality of Care Improvements. This journal is indexed on
18. Denick J, Hu Z, inventors; Bausch \& Lomb Incorporated, assignee Ophthalmic compositions including glycerin and propylene glycol. United States patent US EP0969812A1. 2000 Jan 12.

19. Lonnen J, Heaselgrave W, Nomachi M, Mori O, SantodomingoRubido J. Disinfection efficacy and encystment rate of soft contact lens multipurpose solutions against Acanthamoeba. Eye Contact Lens. 2010; 36(1):26-32.

20. Srivastava DK, Shukla OP. Encystment of Acanthamoeba culbertsoni by organic effectors. Indian J Exp Biol. 1983;21(8):444-447.

21. Kilvington S, Lam A, Lonnen J. Development of a standardised method for the quantified determination of contact lens disinfectant efficacy against Acanthamoeba. Invest Ophthalmol Vis Sci. 2010;51(13): e-abstract 2428.

22. International Organization for Standardization (ISO). International Standard ISO 19045:2015: Ophthalmic optics - contact lens care products - method for evaluating acanthamoeba encystment by contact lens care products. First edition 2015-04-01. Geneva: ISO; 2015.

23. Wilhelmus KR, Osato MS, Font RL, Robinson NM, Jones DB. Rapid diagnosis of Acanthamoeba keratitis using calcofluor white. Arch Ophthalmol. 1986;104(9):1309-1312.

24. Khunkitti W, Lloyd D, Furr JR, Russell AD. The lethal effects of biguanides on cysts and trophozoites of Acanthamoeba castellanii. J Appl Bacteriol. 1996;81(1):73-77.

25. Hamilton MA, Russo RC, Thurston RV. Trimmed Spearman-Karber method for estimating median lethal concentrations in toxicity bioassays. Environ Sci Technol. 1977;11(7):714-719.

26. Cordingley JS, Wills RA, Villemez CL. Osmolarity is an independent trigger of Acanthamoeba castellanii differentiation. J Cell Biochem. 1996; 61(2):167-171.

27. Ahearn DG, Simmons RB, Ward MA, Stulting RD. Potential resistant morphotypes of Acanthamoeba castellanii expressed in multipurpose contact lens disinfection systems. Eye Contact Lens. 2012;38(6): 400-405.

28. Dudley R, Matin A, Alsam S, Sissons J, Maghsood AH, Khan NA. Acanthamoeba isolates belonging to T1, T2, T3, T4 but not T7 encyst in response to increased osmolarity and cysts do not bind to human corneal epithelial cells. Acta Tropica. 2005;95(2):100-108.

29. Kliescikova J, Kulda J, Nohynkova E. Stress-induced pseudocyst formation - a newly identified mechanism of protection against organic solvents in acanthamoebae of the T4 genotype. Protist. 2011;162(1): 58-69.

30. Kliescikova J, Kulda J, Nohynkova E. Propylene glycol and contactlens solutions containing this diol induce pseudocyst formation in acanthamoebae. Exp Parasitol. 2011;127(1):326-328.
PubMed Central and CAS, and is the official journal of The Society of Clinical Ophthalmology (SCO). The manuscript management system is completely online and includes a very quick and fair peer-review system, which is all easy to use. Visit http://www.dovepress.com/ testimonials.php to read real quotes from published authors. 